Variations tibétaines, Et autres...

\title{
ELVERSKOG Johan, Biographies of Eminent Mongol Buddhists
}

PIATS 2006 : Tibetan Studies: Proceedings of the Eleventh Seminar of the International Association for Tibetan Studies, Königswinter 2006, Halle (Saale) : International Institute for Tibetan and Buddhist Studies $\mathrm{GmbH}$ (Beiträge zur Zentralasiatischenforschung; 15) ISBN 978-3-88280-083-8

Isabelle Charleux

\section{OpenEdition Journals}

Édition électronique

URL : https://journals.openedition.org/emscat/1908

DOI : 10.4000/emscat.1908

ISSN : 2101-0013

Éditeur

Centre d'Etudes Mongoles \& Sibériennes / École Pratique des Hautes Études

Référence électronique

Isabelle Charleux, "Elverskog Johan, Biographies of Eminent Mongol Buddhists », Études mongoles et sibériennes, centrasiatiques et tibétaines [En ligne], 42 | 2011, mis en ligne le 20 décembre 2011, consulté le 13 juillet 2021. URL : http://journals.openedition.org/emscat/1908 ; DOI : https://doi.org/ 10.4000/emscat.1908

Ce document a été généré automatiquement le 13 juillet 2021.

(c) Tous droits réservés 


\section{ELVERSKOG Johan, Biographies of Eminent Mongol Buddhists}

PIATS 2006 : Tibetan Studies: Proceedings of the Eleventh Seminar of the International Association for Tibetan Studies, Königswinter 2006, Halle (Saale) : International Institute for Tibetan and Buddhist Studies $\mathrm{GmbH}$ (Beiträge zur Zentralasiatischenforschung; 15) ISBN 978-3-88280-083-8

\section{Isabelle Charleux}

\section{RÉFÉRENCE}

ELVERSKOG Johan, Biographies of Eminent Mongol Buddhists. PIATS 2006 : Tibetan Studies : Proceedings of the Eleventh Seminar of the International Association for Tibetan Studies, Königswinter 2006, Halle (Saale) : International Institute for Tibetan and Buddhist Studies GmbH (Beiträge zur Zentralasiatischenforschung ; 15)

1 Cet ouvrage rassemble neuf contributions présentées lors d'un panel au septième colloque de l'International Association for Tibetan Studies en 2006. Organisé par Johan Elverskog, spécialiste de l'histoire du bouddhisme en Mongolie, ce panel avait pour objectif, au sein d'un colloque de tibétologues, de faire une place nouvelle à la Mongolie dans les études bouddhiques tibétaines. Le bouddhisme mongol, en effet, continue à être qualifié de "tibétain » dans la littérature scientifique, qui semble ne pas vouloir reconnaître un bouddhisme proprement mongol (ou bouriate) - on pourrait par ailleurs ajouter que les nombreuses couches sédimentaires du bouddhisme mongol sont formées d'influences ouïgoures, Xixia, et enfin chinoises. La question se pose de manière d'autant plus sensible que le renouveau du bouddhisme en Mongolie et dans les républiques russes de Bouriatie et de Kalmoukie implique une redéfinition de sa relation au Tibet. L'ouvrage tente également de rendre justice à la grande diversité historique et géographique du bouddhisme mongol : les enjeux religieux, politiques et sociaux de la conversion au bouddhisme et de son rapport aux autorités religieuses tibétaines sont en effet très différents si l'on prend l'exemple d'Altan Qan des Tümed au 
$\mathrm{XVI}^{\mathrm{e}}$ siècle, de la promotion du bouddhisme par les empereurs mandchous au XVII ${ }^{\mathrm{e}}$ et $\mathrm{XVIII}^{\mathrm{e}}$ siècle, des Bouriates du $\mathrm{XX}^{\mathrm{e}}$ siècle dans la sphère russe puis soviétique, ou des renouveaux nationalistes contemporains.

Les contributions, relativement courtes, présentent chacune une figure de l'histoire religieuse de la Mongolie et de la Bouriatie du XVI ${ }^{\mathrm{e}}$ au XXe siècle :

- Neichi Toyin (Nejici Tojin, 1557-1653), célèbre missionnaire torgut qui prêcha dans l'est de la Mongolie-Intérieure,

- Zaya Pandita (Zaja Bandida, 1599-1662), missionnaire oirad qui prêcha parmi les Mongols occidentaux,

- Zanabazar (1635-1723), le premier Jebcündamba Qutu瞵tu,

- Gombojab (Gombužab, fl. 1725-1750), grand traducteur, historien, philologue et médecin dans le Pékin mandchou,

- Agvaandandar (1759-1840), philosophe, linguiste et poète d'Alašan,

- Injannashi (Injannasi, 1837-1892), noble tümed, écrivain, penseur et historien,

- Lunrig Dandar (1831-1920), moine médecin des Qalqa,

- Lubsan Samdan Tsydenov (1850-1922), fondateur d'un état théocratique indépendant bouriate,

- et lama Agvan Nyima (1907-1990), grand philosophe d'origine bouriate.

3 L'introduction nous prévient (p. 10) qu'il s'agit d'un échantillon de moines éminents, et de grandes personnalités ayant eu une contribution majeure dans l'histoire du bouddhisme mongol. D'autres, en revanche, comme le troisième Mergen Gegeen ou Danzin Rabžai n'ont pas été retenues. Ce qui est peut-être dommage: ces deux dernières incarnations furent des réformateurs, porteurs d'un bouddhisme fondamentalement mongol, ce qui n'est pas le cas de certains grands lamas tibétanisés au point qu'on les croit souvent d'origine tibétaine (comme Agvan Nyima). À ce sujet, on consultera la thèse très riche, en cours de publication, de B. Uranchimeg ${ }^{1}$, centrée sur Neichi Toin et Mergen Gegeen, qui ont véritablement inventé un bouddhisme mongol, et en langue mongole. En retour, certains de ces grands lamas tibétanisés ont eu un impact certain sur le bouddhisme au Tibet.

4 Certains d'entre eux ne nous sont connus que par leur biographie (comme Zaya Pandita et Neichi Toin), tandis que d'autres ont laissé maints écrits, œuvres artistiques et témoignages variés (Gombujab, Agvaandandar, Agvan Nyima). Les trois premiers articles biographiques sont fondés sur des rnam-thar, biographies écrites en tibétain, en mongol ou dans les deux langues, employées comme « a vehicle to reveal the dynamic nature of Buddhism among the Mongols » (p. 9). Ils donnent des informations sur leur auteur, la date de la composition, le style littéraire, les différentes éditions des biographies, et leurs traductions éventuelles. Heureusement pour les historiens, ces biographies ne sont pas seulement des hagiographies à la structure littéraire formatée, même si souvent elles en reprennent le modèle : celle de Neichi Toin par exemple est narrative et met l'accent sur les pratiques et les rituels plutôt que sur les concepts abstraits ; c'est plutôt sur la biographie de Milarepa, traduite en mongol au XVII ${ }^{e}$ siècle, qu'elle prendrait modèle (p. 18). Celle de Zaya Pandita est quant à elle originale, puisqu'elle ressemble plus à une chronique historique, évoquant les conflits mongolsoirad. Par contraste, quelques grands savants dont la biographie n'a pas été retrouvée ou écrite, ne nous sont connus que par leurs écrits et la tradition orale, comme Gombojab et Agvaandandar. 
Ces grandes figures religieuses ayant déjà fait l'objet de nombreuses études, les articles visent en général ici à corriger certaines erreurs répandues et à apporter des compléments d'informations. Karénina Kollmar-Paulenz ("Forming a Mongolian Buddhist Identity: The Biography of Neichi Toin”, pp.13-28) montre par exemple l'erreur d'interprétation que Walther Heissig commit dans sa remarquable étude de la biographie mongole du Premier Neichi Toin, grand missionnaire du début du $\mathrm{XVII}{ }^{\mathrm{e}}$ siècle. En effet, bouddhisme et chamanisme ne peuvent être vus comme deux systèmes religieux institutionnels opposés. Ce sont exclusivement les chamanes (par essence non institutionnalisés et non organisés) en tant que spécialistes religieux, et leurs dangereux ongod (figurines représentant des esprits chamaniques) que Neichi Toin s'acharne à détruire, et nulle part dans sa biographie n'est fait mention d'une opposition quelconque au culte aux divinités du terroir (gazar-un ezen), aux ovoo ou au Ciel. (Pour une étude approfondie de la biographie de Neichi Toyin, on consultera la thèse de B. Uranchimeg citée ci-dessus). Les moines bouddhistes n'ont pas cherché à substituer le bouddhisme aux pratiques populaires, mais à fournir des interprétations nouvelles aux pratiques religieuses. Neichi Toin accusa les chamanes et chamanesses

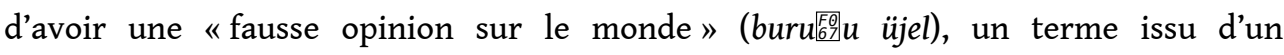
vocabulaire bouddhique ancien ayant servi d'argumentaire contre le système religieux tibétain pré-bouddhique (le chos-log, « faux dharma »).

6 Hidehiro Okada et son épouse Miyawaki-Okada Junko ("The Biography of Zaya Pandita, the Greatest Oirad monk », pp. 29-47) montrent que la biographie de Zaya Pandita des Oirad ne parle en aucun cas d'un "khanat» oirad, conception erronée qui a été propagée par Zlatkin (Istoriia Dzhungarskogo Khanstva (1635-1757), 1964) : les Oirad étaient organisés en une confédération dirigée par des chefs zungar. La biographie, écrite en " écriture claire » inventée par Zaya Pandita, fut par la suite retranscrite en écriture ouïgouro-mongole, et est en cours de traduction et annotation en japonais par Miyawaki-Okada Junko.

7 Se fondant sur la biographie de Zanabazar par Luvsanprinlei, le Zaya Pandita des Qalqa, Agata Bareja-Starzyńska ("The growth of the religious authority of the First Jebdzundampa of Mongolia (1635-1723)», pp. 49-57) reprend des questions posées précédemment par Miyawaki Junko et d'autres chercheurs, en particulier la date et la raison de la reconnaissance de Zanabazar en tant que réincarnation ou émanation du grand polymathe tibétain Tāranātha (1575-1635). Ce dernier, en effet, appartenait à la tradition Jonangpa, persécutée par le cinquième Dalaï-lama: pourquoi le cinquième Dalaï-lama a-t-il donc reconnu Zanabazar comme réincarnation d'un grand lama d'une école bannie? Le paradoxe d'une réincarnation jonangpa devenue le principal hiérarque des Mongols intrigua également ses contemporains. A. Bareja-Starzyńska prépare une traduction de la biographie de Zanabazar par Luvsanprinlei, écrite en tibétain et traduite en mongol.

8 Johan Elverskog («Injannashi, the Anti-Cervantes », pp. 83-106) présente une figure bien connue de la littérature mongole, Injannashi. On peut être surpris de son inclusion dans ce volume, puisque Injannashi non seulement est un laïc, mais encore est réputé pour ses écrits anticléricaux voire anti-bouddhiques. J. Elverskog souligne tout l'intérêt d'étudier l'œuvre d'Injannashi dans sa globalité - son histoire des Mongols, le Köke sudur, mais aussi les nouvelles sinisantes dans la continuité du «Rêve dans le pavillon rouge " (Honglou meng) - et le compare ici à la figure diamétralement opposée de Cervantès. Contrairement à ce que l'on retient si on ne lit qu'une portion de son œuvre, 
Injannashi n'était pas un novateur mais un conservateur élitiste et éclectique, qui cherchait à sauver l'ordre aristocratique gengiskhanide déclinant, qu'il considérait comme seul légitime. S'il empruntait le style des lettrés chinois, ce n'était que pour réaffirmer la suprématie de la noblesse. Il n'était donc pas un proto-révolutionnaire nationaliste ou encore un pro-confucéen faisant l'apologie de l'empire Qing.

Les cinq autres articles sont moins polémiques vis-à-vis de l'historiographie existante, et font le point sur la vie et l'œuvre de personnalités religieuses et s'intéressent plutôt à l'histoire intellectuelle. Vladimir Uspenskiy («Gombojab - A Tibetan Buddhist in the capital of the Qing empire ", pp. 59-69) énumère les œuvres de Gombojab conservées dans les canons chinois, tibétain et mongol: manuel d'iconographie, traduction de romans chinois, historiographie du bouddhisme, dictionnaires... écrits aussi bien en mongol qu'en tibétain. L'« Histoire du bouddhisme en Chine » (Rgya nag chos 'byung) de Gombojab, écrite en 1736, fut pendant longtemps la principale source concernant l'histoire de la Chine disponible pour les lecteurs tibétains. V. Uspenskiy montre que Gombojab fut un pionnier en matière de philologie, d'ethnogenèse et d'histoire, même si ses théories, encore reprises par des historiens mongols contemporains, sont parfois erronées. C'est ainsi que des historiens continuent d'affirmer que les Xiongnu furent les ancêtres des Mongols, et furent convertis au bouddhisme avant les Chinois. (La bibliographie de V. Uspenskiy peut être complétée par l'article de Françoise WangToutain, «Circulation du savoir entre la Chine, la Mongolie et le Tibet au XVIII ${ }^{e}$ siècle. Le prince mGon-po skyabs », Études Chinoises XXIV (2005/2006), pp. 57-111).

10 Magdalena Szpindler ( Agvaandandar (1759-1840) - Philosopher, linguist and poet from Alashan», pp.71-82) résume ce que l'on sait de la vie et de l'œuvre d'Agvaandandar, polymathe comparable aux savants de la Renaissance, et bien connu des tibétologues pour son dictionnaire et ses œuvres de logique, de grammaire et de poésie. Un colloque qui s'est récemment tenu en Alašan suivi de trois volumes d'actes montre la vitalité des études sur ce grand savant dont l'œuvre est publiée dans tout le monde tibétain, de New Delhi à la Bouriatie.

11 D. Ganzorig («Lunrig Dandar: Life and works of one of the last great scholars of Tibetan medicine in Mongolia ", pp. 107-116), qui lui-même fit des études de médecine tibétaine à Dharamsala, retrace les grandes lignes de la biographie (les études, les maitres, les diplômes et les monastères dans lesquels il étudia) et les nombreux écrits du grand médecin Lunrig Dandar de la province Sajin Nojan Qan. L'auteur participa à une expédition du Centre Mongol Bilig pour l'Étude des Ouvrages Tibétains Écrits par des Mongols, et découvrit en 2004 un texte rituel manuscrit précédemment inconnu de Lunrig Dandar, sur le culte de la constellation de la Grande Ourse.

12 Nikolay Tsyrempilov («Samdan Tsydenov and his Buddhist theocratic project in Siberia », pp.117-138), présente des éléments de la biographie de Lubsan Samdan Tsydenov (1850-1922), polymathe, ascète bouddhique, abbé du monastère de Kudun et poète religieux, qui fonda en 1919 un état théocratique indépendant dans une enclave du territoire bouriate, basé sur une conception bouddhique de l'autorité. Tsydenov obtint le support des nobles et du clergé (et aussi du peuple cherchant à éviter la conscription, alors très impopulaire), et fut proclamé Dharmarāja des trois mondes et détenteur de l'autorité religieuse et civile. Son état théocratique pacifiste, condamné à l'échec dans le contexte politique de l'époque, fut écrasé par l'Armée rouge au début des années 1920. L'auteur tente d'expliquer les différents témoignages concernant le refus de Tsydenov de se prosterner lors de l'intronisation du Tsar Nicolas II, et traduit 
pour cela le complexe poème d'éloge au Tsar qu'il rédigea en tibétain : Nicolas II y est présenté comme un souverain cakravartin régnant sur une Terre Pure, certes non bouddhique mais porté par une morale universelle équivalente au Dharma. Tsyrempilov détaille la fascination de Tsydenov pour l'Europe, et montre que ce sont bien les systèmes politiques occidentaux, et en particulier la démocratie, et non les théocraties mongoles et tibétaines, qui ont inspiré la forme d'autorité qu'il mise en œuvre.

13 Natalia Bolsokhoeva («Lama Agvan Nyima (1907-1990) - The Tibetan Buddhist Master from Buryat-Mongolia ", pp. 139-147) présente la biographie d'Agvan Nyima, grand savant qui reçut une éducation bouddhique classique à Gomang (collège monastique du monastère de Drepung qui accueillait particulièrement les moines mongols), s'exila en 1958 en Inde, puis fut invité à résider aux Pays-Bas, au Canada et en Suisse. De 1977 à 1980, il fut abbé de Gomang, monastère reconstruit en Inde du Sud. À tous les points de vue, ce grand expert en philosophie bouddhique, auteur de nombreuses œuvres en tibétain, dont une autobiographie et un ouvrage en seize volumes sur les biographies de grands maîtres bouddhiques, peut être considéré comme un grand maître tibétain. Bien que né en Bouriatie, il passa 67 années hors de son pays et n'y retourna jamais. Pourtant ses disciples bouriates, formés en Inde du Sud, entretiennent sa mémoire dans son pays natal.

14 Cet ouvrage, qui oscille entre particularisme du bouddhisme mongolo-bouriate et « universalisme » du bouddhisme tibétain sera utile à tout chercheur travaillant sur le bouddhisme tibéto-mongol. Il a le mérite de faire connaître dans le monde anglophone des travaux de chercheurs russes, mongols et japonais.

\section{NOTES}

1. Uranchimeg Borjigin Ujeed, "Indigenous Efforts and Dimensions of Mongolian BuddhismExemplified by the Mergen Tradition", PhD dissertation, SOAS, Department of the Study of Religions, Londres, 2009. 